\section{Implants: techniques for fitting and removal}

The two letters ${ }^{1} 2$ describing individuals' different techniques for implant removal that have appeared in recent issues of this Journal are timely. Routine removal with or without refit is common practice throughout the UK as implants have become widely available and popular. It is not yet clear whether introduction of Nexplanon ${ }^{\circledR}$ (MSD, Hoddesdon, UK) will lead to fewer difficult removals. It is evident from the aforementioned letters and conversations between clinicians that diverse and innovative approaches have evolved for fit and removal. These letters are therefore relevant as they highlight the need to recognise key principles to follow when removing (and fitting) implants. Whatever the technique it should be minimally traumatic/invasive, effective and - importantly - safe for the patient and safe for the operator. Dr Menon questions the merit of Dr Kandiyil's splinting technique for routine removal. He points out that blind introduction of the needle theoretically and unnecessarily could damage deeper structures. I would be interested to learn, in terms of these principles, what comparative advantages Dr Kandiyil's method has over the simple use of local anaesthetic and scalpel for palpable devices.

Removal of impalpable implants presents a different situation and this same technique, when used with ultrasound guidance, minimises risk to otherwise unseen structures and focuses the operating field on the precise location of the implant. It also has the advantage of bringing the implant superficially towards the skin where it is then often palpable. Ultrasound-guided splinting technique therefore provides safety advantages for the patient. Having used this method for a few years I have, however, faced the challenge of identifying a technique that is 'safe' for the operator (and have for this reason been reluctant to teach the method to others). Use of a regular phlebotomy needle puts the operator at risk of injury. I have considered various needles (amniocentesis, spinal, etc.) looking for combined properties of slim but firm, suitable to penetrate arm skin, and long enough that the exposed end can be safely sheathed while operating. Most recently the Steritex ${ }^{\circledR}$ V-Air vent needle (Steritex, Göttingen, Germany) has proved to be a satisfactory solution.

I am keen to learn what other devices clinicians have found safe and suitable for this purpose.

Splinting is just one novel practice that has been adopted. The following are examples of other developments:

- Bandages are no longer applied following fitting or removal

- Fitters no longer anaesthetise along the insertion track

- Ethyl chloride is an alternative to lidocaine for fitting (not removal).

MSD convenes implant-fitting workshops but there has been to my knowledge no formal arrangement for appraisal of practice. I wonder whether it is time as a specialty for a process of bringing together experiences and methods, complete with success rates, complications, advantages/disadvantages and patient feedback, with the intention of improving practice and informing training for the future. I look forward to hearing Journal readers' views on this.

\section{Julia Shefras, MRCOG, MFSRH}

Consultant in Contraception and Sexual Health, Alec Turnbull Centre, Oxford Health NHS Foundation Trust, Cowley, Oxford, UK;

julia.shefras@oxfordhealth.nhs.uk

Competing interests None.

J Fam Plan Reprod Health Care 2013;39:65. doi:10.1136/jpprhc-2012-100494

\section{REFERENCES}

1 Kandiyil VN. Easy method for Implanon ${ }^{\circledR}$ removal. J Fam Plann Reprod Health Care 2012;38:207-208.

2 Menon K. Implanon ${ }^{\circledR}$ removal technique. J Fam Plann Reprod Health Care 2012;38: 271-272. 